The Patrent.

It is not fit that we medical men should be accused of treating the human body as if it were merely so many cubic centimetres of material. We are all conscious of the so-called "personal element" - that tantalizing something - present, but yet not measurable. A patient for radiation should not only be thoroughly investigated physically, but his age, state of health, and so on, must be taken into consideration before deciding whether radiation will achieve anything beneficial to him.

Radiation being decided upon, the next point is to map out clearly the lines of treatment to be adopted. These may be palliative or curative. Of the patients seen by radiologists, in the majority palliation only is all that can be hoped for. This side of radiotherapy is of great value, but is being lost sight of in our efforts to cure. It is quite impossible, in certain cases, to attempt to eradicate the disease, and the chances are that if we do not discriminate the last condition of the patient will be worse than the first. The therapeutic goal varies, and each case must become a study in itself. I make it a rule to put down the objects I hope to achieve by radiation, and my plan of treatment is arranged accordingly.

Radiation Sickness or Malaise.-Radiation sickness is the bugbear of radiatic".. We have to inquire what its cause is, and whether it should be regarded as evidence of overdosage. The results are often disastrous to the patient, being sufficient to turn the scale against him. This puzzling phenomenon may manifest itself in several ways. Actual vomiting may occur as the patient is undergoing treatment, or sudden nausea with or without vomiting may be experienced from one to seventy-two hours after. As hospital patients describe it, "I come all over alike-suddin'." Intense headache may be complained of without gastric symptoms. With the high-voltage line of treatment vomiting may become uncontrollable. In its treatment various drugs have been advocated from time to time, but so far I have found nothing to control it. At present I do not consider that we are justified in subjecting a patient to the risk of severe radiation sickness unless we can promise him something substantial in return.

\section{Carcinoma Mammae.}

The majority of cases that find their way to the radiological department of the London Hospital, and probably at most of the hospitals in England, come under this heading. Over two thousand have been treated at the London Hospital in the last fourteen years. For this reason I have always endeavoured to improve the technique for this large class of case. Whether inoperable, recurrent, or requiring prophylactic treatment, malignant disease of the breast illustrates very clearly the problem we have to solve in radiation of cancer.

Prophylactic Methods of Radiation.-After the primary growth has been removed, not by radiation, but by the surgeon, the radiologist is called in. His interest now is not distracted by the presence of a tumour; he has to devise a technique that has a reasonable chance of destroying cells at unknown depths and positions. This is a complex problem, but it must be faced. The surgeon can, in a number of cases, remove the primary growth and glands without our help, but he is not satisfied with the results because he does not necessarily prevent metastases or recurrence. This, then, is where the radiologist can help. Even if it were possible to destroy with certainty every primary growth by radiation or any other method, I personally should not be satisfied. Metastases kill the patient just as rapidly. We must control these in addition to the original cancer.

I consider heterogeneous radiation to be of greater therapeutic value than homogeneous in the prophylactic treatment of breast cancer, for these reasons: It gives radiation absorption at various depths; a large radiation field can be utilized with minimum general disturbance. I readily grant that further development is required on these lines. I have already reached the limit of the toleration of the patient under these conditions.
There are four chief objections to the high-voltage method in this class of case :

1. The radiation field is necessarily much too limited.

2. There is no indication as to the depth at which the dose should be delivered.

3. Damage to the lung tissue is comparatively frequent.

4. General disturbance-such as sickness and blood changes-place too great a strain on the patient.

The response of many a hopeless case is often remarkable if the heterogeneous radiation is carefully carried out.

Radiation of the Whole Trunk.-I maintain that whatever form of radiation is used in cases of breast carcinoma, it is useless and against common sense to limit it to local areas. Radiation should, if possible, include the whole trunk. It benefits the patient but little to check the metastases in a localized area while they go ahead elsewhere. As there is also evidence for the theory that in many cases the patient's tissues-we do not know which-have the ability of producing some beneficial reaction, the larger the field of radiation the better.

As a preliminary effort towards achieving the conditions I consider necessary in the treatment of these cases, I introduced the dual open method at the London Hospital some three years ago. Briefly stated, it consists in placing a tube at the back and front of the patient, both running at the same time, the radiation field including practically the whole trunk. The results show, without doubt, that this arrangement has greatly increased our range of control, benefit being now obtained in cases previously out of our reach, such as secondary deposits in the spine. This is all to the good, but much research is necessary before any further advance can be expected. To my mind this is certainly a logical method of radiation, where stray pathological cells may be at unknown depths and positions. If must be realized that attempts to substitute physics for biology and medicine can only hinder sound progress in radiotherapy.

\section{EMPYEMA OF THE LEFT SPHENOIDAL SINUS WITH OPTIC NEURITIS ANU SUBSEQUENT POST-PAPILLITIC ATROPHY BY}

R. E. WRIGHT, M.D., MAJor I.M.S., SUPERINTENDENT, GOVERNMENT OPHTHALMIC HOSPITAL, MADRAS.

WithodT actual exploration of the sphenoidal sinuses it is impossible to exclude disease of these cells as the responsible factor in certain cases of optic nerve disease. A very considerable amount of work has been published on this subject, but in spite of careful observations we are still without reliable guides, either signs or symptoms, to lead us to a definite diagnosis in cases of optic neuritis due to sphenoidal sinusitis. The case here recorded is an example of one in which there was little to indicate the true nature of the case; it also emphasizes the value of a routine examination of the sinuses on both sides.

A Hindu woman, aged 40, married, was brought to see me on April 17th, 1923, complaining of loss of vision in the right eye and lieadache of two month's duration. The vision of the right $\epsilon y \theta$ was fingers at 3 metres; left eye vision $6 / 9$ bar one letter with plus 1.0. D.S. $=6 / 5$. The headache was frontal for the most part, but with definite occipital pain, chiefly on the right side. The pupil reactions and tension were normal on either side. The right disc was somewhat swollen and oedematous on the nasal side, pallid and steamy in appearance; the arteries were narrowed and sheathed (subsiding papillitis). The left disc showed narrowed and sheathed (subsiding papillitis). The left disc showed quiet neuritis, a red swollen disc with definite venous engorgement.
The eye grounds were otherwise normal. There was no history of syphilis or evidence of it clinically; the Wassermann test was negative. There were no symptoms pointing to cerebral disease except headache. The reflexes were normal, as also the urine and blood. There was slight pyorrhoea, but $x$-ray photographs did not show anything definite either as regards teeth or sinuses. The right field could not be taken; the left was examined with Lister's perimeter in uniform daylight illumination (intense Lister's perimeter in uniform daylight illumination (intense
north light over each sbulder). $5 \mathrm{~mm}$. at $33 \mathrm{~cm}$. for white and 
colours, and was well within normal limits. Scotometric tests with Bjerrum's screen and Elliot's scotometer revealed nothing abnormal, either as regards scotomata, relative or otherwise, with white or colours, or enlargement of the blind spot.

The patient was temporarily put on mercury and large doses of potassium iodide. As a routine I would have examined the nasal potassium on obtaining such negative results, but unfortunately sinuses on obtaining such negative results, but unfortunately
I left for Europe on April 24th, 1923, and she went home and continued to take mercury and potassium iodide. On January 3 rd, 1924, the Wassermann reaction was observed to be negative. On July 3rd I again had an opportunity of examining her. Vision was then: right, fingers at 2 inches; left, $6 / 5$ partly with correction. There was definite post-papillitic atrophy of the right disc with narrowing of vessels. The left disc was oedematous and swollen to $5 \mathrm{D}$. (suggestion of papilloedema). Examination of and swollen to $\mathrm{D}$. (suggestion of papilloedema). Examination of
blood, urine, nervous system, and skul by $x$ rays gave negative
results. There were no symptoms of pressure. The fields and vision of the left eye remained the same. I was unable, owing to indisposition, to undertake the exploration of the sphenoidal cells at this time. She went to her home and was not seen again till August 13th, when she was admitted to hospital. The result of ophthalmoscopic examination on this date is thus recorded: "The right disc is definitely atrophic. The arteries are quite narrow, the veins about normal; there are slight pigmentary disturbances at the disc edge; a post-papillitic atrophy. The left disc is red and slightly. swollen, but much more like a neuritis than a papilloedema. There are no changes in the fundus otherwise." Perimetric and scotometric examinations gave the same results as before; the urine and blood examinations were again negative. The blood pressure was $125 \mathrm{~mm}$. Hg.

On August 16th I made a full examination of the nose on both sides under local anaesthesia. The right side was normal. The sphenoidal sinus ostium when opened up disclosed a pearly-grey healthy membrane lining a small sinus. The left side of the nose appeared normal as regards frontal and ethmoidal regions, but, the ostium of the sphenoidal sinus was blocked, and, on pushing the sinus probe through, pus-escaped under slight pressure along the probe. The anterior wall of the sinus was partially removed, revealing a thickened and red lining. The sinus was dried out and packed with bismuth gauze and the patient sent for $x$-ray examination.

This method of demonstrating the relation of one sphenoidal sinus to its fellow by packing one sinus with bismuth gauze and then taking a stereoscopic $x$-ray photograph is useful, and aids considerably in reading the $x$-ray plates aright. The left sinus was much more extensive than the right, and partially enveloped it. Presumably it was of the variety to which both nerves were related, the right nerve being more vulnerable (either by virtue of a larger course in the sinus or a thinner bony canal) than the left. Such relations of the optic nerves are described and exemplified by Onodi. The patient was dicharged a few days later. In November, 1924, the vision was: right, fingers at 2 metres; left, 6/6 with correction. The left field showed a slight general contraction under the same conditions as above. On ophthalmoscopic examination the left disc was almost normal in appearance. The right disc showed a post-papillitic atrophy with threadlike sheathed arteries. My note says: "The fundus immediately surrounding the disc shows a greyish granular appearance, but I would consider the disc within normal limits did I not recognize the patient."

A most interesting point in connexion with the case is the nature of the field of the left eye. Careful tests on several occasions elicited nothing. This is in contrast with many recorded cases, in which a relative central or paracentral scotoma is a prominent feature. The fact that the right nerve suffered most in a left sinus affection must be comparatively uncommon. The left nerve, although affected in greater or lesser degree for over eighteen months, retained its function (although subsequent subsidence of its inflammatory condition brought as a sequel a slight cicatricial damage associated with a trivial fall in vision and a contraction of the fields). It is noteworthy that in an ordinary $x$-ray examination pus in the sphenoidal sinus is liable to be overlooked. Evidently, in doubtful cases of papillitis, it is very desirable to explore the sphenoidal sinuses on both sides; one must not be content with finding a normal sinus on the side of the most affected eve. This is in many cases such a simple procedure that there is no justification for omitting it.

Dr. Muthayya, medical officer in charge of the refraction department, very kindly collected and analysed the notes of this case.

USEFUl REFERENCES.

American Encyctopedia of Ophthalmology, vol. xii, p. 9588.

Onodi : The Ortic Nerve and the Accessory Sinuses of the Nose (translation by Lückhofi).

\section{EMBRYOMA IN THE OMENTUM CAUSING SYMPTOMS SUGGESTIVE OF URETERAL CALCULUS.}

BY

\section{A. E. WEBB-JOHNSON, C.B.E., D.S.O., M.B.,} F.R.C.S.,

SURGEON TO THE MIDDLESEX HOSPITAL.

THE following case is of interest, both from the point of view of pathology and of diagnosis.

A woman, aged 40 , was sent to see me at the Middlesex Hospital, with a provisional diagnosis of renal calculus. Her symptoms were certainly suggestive of this condition being present. She complained of attacks of pain in the left loin coming on suddenly and radiating towards the inguinal region. coming on suden alder with She also complained of some irritability of the bladder with painful and frequent micturition, which persisted for some time after the attacks of lumbar pain. The urine contained pus, but no blood communis.

An $x$-ray examination showed the kidney region to be free from any sign of calculus, but in the pelvis was a shadow of irregular shape, about the size of a large plum-stone. Cystoscopic examination showed the bladder and the ureteral orifices to

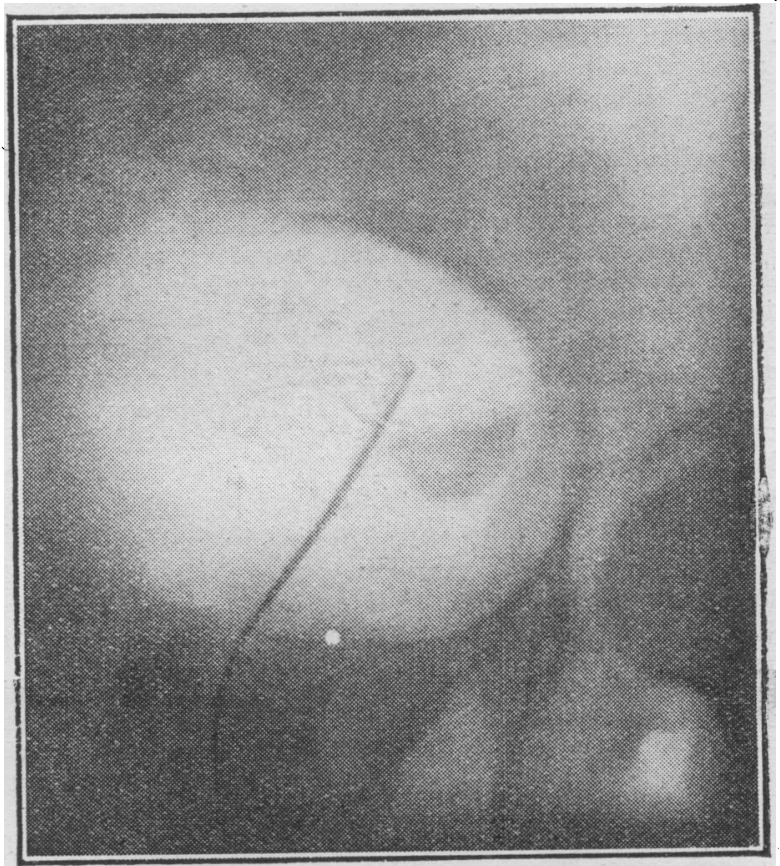

Fic. 1.-Radiogram taken in an oblique direction with an opaque catheter in the ureter.

be normal, with the exception of some slight congestion of the bladder mucous membrane. Although the shadow in the pelvis was not in the least like that of a urinary calculus, I thought it better to make sure that it was not in the urinary tract. I therefore passed an opaque ureteral catheter, and had an $x$-ray photograph taken. The examination showed that the shadow of the catheter was superimposed on that in the pelvis, but another radiogram taken in an oblique direction show'ed that the shadow was separated by a small interval from the course of the ureter (Fig. 1). As the irregular shape of the shadow suggested that it (Fig. 1). As the irregular shape of the shadow suggested that it might be caused by a calcareous deposit in an ovary, I asked Mr. Comyns Berkeley to co-operate with me in an exploratory
laparotomy. Mr. Berkeley opened the abdomen, but found that both ovaries were normal; I then extracted a small, hard irregular-shaped tumour, freely movable across the pelvis, but attached above to the lower edge of the omentum and below by a thin fold of peritoneum to the posterior wall of the bladder. The connexion with the bladder and the free mobility of this hard mass probably accounted for the patient's symptoms of hard mass probably accounted for the patient's symptoms of pain and frequency of micturition,

On section the tumour was found to be of mixed formation, part of it being cystic and part of it hard, bony, and calcified. The cystic part contained a mass of hair and sebaceous matter. The tumour was evidently a small embryoma.

In Sir John Bland-Sutton's book Tumours, Innocent and Malignant, we read:

"Many cases have been reported in which cysts containing pilose skin, and occasionally teeth, have been found on the omentum and the serous covering of the intestine. In some 